\title{
Magnetic resonance diffraction using the magnetic field from a ferromagnetic sphere
}

\author{
Mladen Barbic ${ }^{\text {a) }}$ \\ Department of Applied Physics M/S 200-36, California Institute of Technology, Pasadena, California 91125
}

(Received 4 January 2002; accepted for publication 2 April 2002)

\begin{abstract}
The theory of magnetic resonance diffraction is developed for the case of a crystal in close proximity of a ferromagnetic sphere. Distinct spectral peaks in the magnetic resonance signal are discovered for the specific ferromagnetic sphere and magnetic field configurations, and the appearance of the peaks is a direct signature of the presence of discrete atomic sites in the crystal lattice. The positions of the spectral peaks are sensitive to the crystal unit-cell size, thereby providing a method for determination of the basic parameters of the crystal at the atomic scale. The appearance of the spectral peaks is explained, and the dependence of the magnetic resonance spectra on the sphere size and the angle of the sphere magnetization with respect to the sample surface is analyzed. Applications to the studies of crystals, thin films, and crystallites are reviewed, and potential measurement methods for the confirmation of the diffraction theory are proposed. The analysis suggests that the long-desired goal of detecting atomic resolution magnetic resonance diffraction is well within reach of current experimental techniques. (C) 2002 American Institute of Physics. [DOI: 10.1063/1.1480466]
\end{abstract}

\section{INTRODUCTION}

In 1973, two independent and pioneering papers by Lauterbur $^{1}$ and Mansfield and Grannell ${ }^{2}$ reported on applying magnetic field gradients to samples in order to demonstrate magnetic resonance imaging of spatial spin distribution. Since then, magnetic resonance imaging (MRI) has become a well-established three-dimensional (3D) visualization technology with tremendous impact on clinical medicine. ${ }^{3}$ Although improvements in imaging resolution have steadily progressed in the last three decades, the current spatial resolution is limited to approximately $1 \mu \mathrm{m}^{4-6}$ due to the low energy photons involved in the magnetic resonance process. ${ }^{7}$ This is contrasted by other imaging technologies, such as electron microscopy ${ }^{8}$ and various scanning probe microscopies, ${ }^{9,10}$ that have provided atomic resolution. Scanning probe techniques have also been extended to surface atomic resolution magnetic imaging ${ }^{11}$ and magnetic resonance spin imaging. ${ }^{12}$

Motivated by the potential of combining 3D imaging capability of conventional magnetic resonance and the atomic resolution of scanning probe techniques that utilize mechanical cantilevers, Sidles proposed a unique atomic resolution 3D magnetic resonance imaging technique. ${ }^{13}$ This method, magnetic resonance force microscopy (MRFM), uses a microscopic magnetic particle as a source of atomic scale imaging gradient fields and a mechanical resonator as a sensitive detector of magnetic resonance. ${ }^{14}$ This instrument marks a change in direction in magnetic resonance detection $^{15}$ from the standard inductive techniques. ${ }^{16,17}$ Proof-of-concept demonstrations of the technique were carried out for various magnetic resonance systems including

a) In 1999 the author changed his last name from Todorovic to Barbic; electronic mail: mladen@caltech.edu electron spin resonance, ${ }^{18}$ nuclear magnetic resonance, ${ }^{19}$ and ferromagnetic resonance. ${ }^{20}$ While this technique is rapidly progressing by the incorporation of smaller magnetic particles $^{21,22}$ and more sensitive mechanical resonators, ${ }^{23}$ current MRFM imaging resolution of $\sim 1 \mu \mathrm{m}^{24,25}$ remains at the level of conventional MRI inductive detection.

While achieving single-spin sensitivity and resolution in a 3D imaging technique is of great significance, the MRFM technique also places challenging demands on the technical requirements, such as operation at very low temperatures, miniaturization of mechanical cantilevers, and the integration of magnetic nanoparticles into resonating structures. It is an intriguing question whether the method currently pursued for achieving single-spin sensitivity and resolution can be complemented by a similar approach that relaxes the MRFM sensitivity requirements by allowing many spins to coherently contribute to the magnetic resonance signal while still providing atomic scale information. This was the initial goal of the seminal work by Mansfield and Grannell ${ }^{2,26}$ who introduced linear field gradients to resolve the atomic lattice planes of the crystal. Their proposed magnetic resonance diffraction technique resembles several other analytical techniques such as X-ray diffraction, ${ }^{27}$ neutron diffraction, ${ }^{28}$ and electron diffraction, ${ }^{29}$ which rely on the concept of collecting a signal from many atomic sites, but reveal the atomic scale information from the sample due to the regular arrangements of the crystal atoms and the wave nature of the probing beam. These analytical methods result in signal peaks at unique scattering angles from which atomic scale information about the crystal can be deduced. In this article, the numerical prediction of sharp magnetic resonance diffraction peaks from a crystal in proximity of a microscopic ferromagnetic sphere is reported. These peaks represent a direct signature of the regular atomic structure of the crystal, and their detection could lead to the realization of the long sought- 


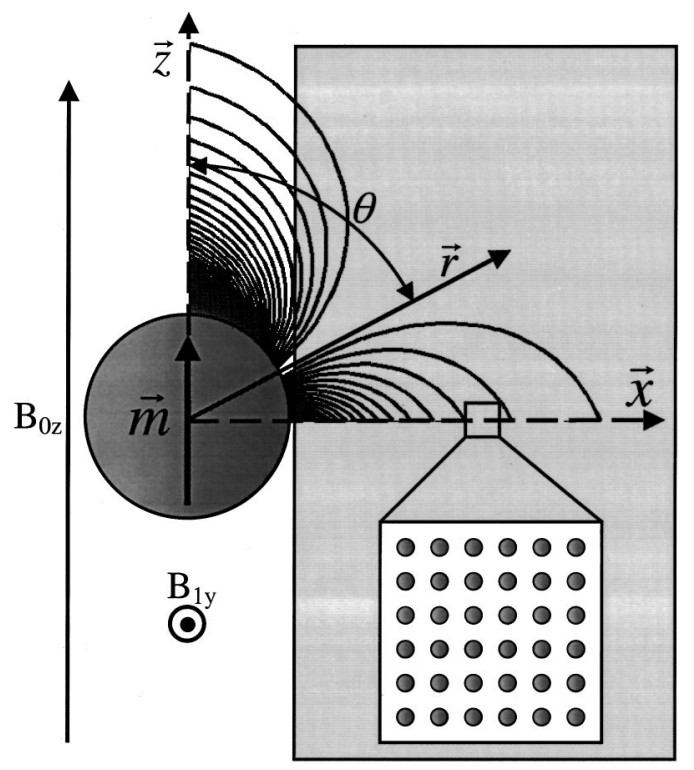

FIG. 1. Configuration for the magnetic resonance diffraction using the field from a ferromagnetic sphere. Large polarizing magnetic field $B_{0}$ is applied parallel to the sample surface. It polarizes the sample spins and saturates the ferromagnetic sphere magnetization. Small radio-frequency field is applied perpendicular to the polarizing field for magnetic resonance investigation. Contours of constant $B_{Z}$ are also indicated in the figure, and they have the azimuthally symmetric form around the $z$ axis. Discrete nature of the atomic sites in the sample is explicitly introduced into the model.

after goal of atomic scale magnetic resonance diffraction.

\section{MAGNETIC RESONANCE DIFFRACTION MODEL}

The basic model configuration is illustrated in Fig. 1. A ferromagnetic sphere is in proximity of the surface of a simple cubic lattice crystal. The sphere is assumed to be 100 $\mathrm{nm}$ in diameter and made of cobalt with the magnetization per unit volume of $1500 \mathrm{emu} / \mathrm{cm}^{3}$. The crystal is assumed to have a unit-cell size of $a_{0}=3 \AA$. A large DC magnetic field $B_{0}$ is applied parallel to the sample surface, with the purpose of polarizing the spins of the atomic lattice for magnetic resonance investigation, as well as saturating the magnetization of the ferromagnetic sphere. A small radio-frequency field $B_{1}$ is applied perpendicular to the large polarizing DC magnetic field $B_{0}$. In the absence of the ferromagnetic sphere, the atomic spin sites in the crystal would experience the same externally applied field $B_{0}$ and therefore meet the magnetic resonance condition at the same magnetic resonance frequency $\omega_{R}$. Close to the ferromagnetic sphere, a large magnetic field gradient is present within the crystal, and only certain spin sites of the lattice satisfy the correct magnetic resonance conditions at any given magnetic field and frequency. Standard convention is taken that the large external DC magnetic field is applied in the $z$ direction. The magnetic field from the ferromagnetic sphere at point $r$ in the sample has the azimuthally symmetric dipolar form

$$
\vec{B}(\vec{r})=\frac{3 \vec{n}(\vec{m} \cdot \vec{n})-\vec{m}}{|\vec{r}|^{3}}
$$

where $n$ is the unit vector that points from the center of the ferromagnetic sphere to the crystal site location, and $m$ is the magnetic moment vector of the sphere. Since the external DC polarizing magnetic field $B_{0}$ is considered to be much larger than the field from the ferromagnetic sphere, of $B_{0}$ $\sim 10 \mathrm{~T}$, only the $z$ component of the magnetic field from the ferromagnetic sphere is included in considering the resonant spins of the atomic lattice

$$
B_{Z}(\vec{r})=\frac{M_{0}}{|\vec{r}|^{3}}\left(3 \cos ^{2} \theta-1\right),
$$

where $\theta$ is the angle between the $z$ axis and the distance vector $r$ as shown in Fig. 1, and $M_{0}$ is the magnitude of the saturation magnetic moment of the ferromagnetic sphere. The contours of constant field $B_{Z}$ from the sphere are also shown in Fig. 1, and they have the azimuthally symmetric form around the $z$ axis.

If the ferromagnetic sphere is sufficiently small that the magnetic fields vary strongly on the atomic scale, steps need to be taken into account for the discrete nature of the crystal lattice. Labeling the atomic sites with indices $(m, n, l)$, and assuming a unit-cell size with dimension $a_{0}$, the components in expression (2) take the form

$$
\begin{aligned}
\cos ^{2} \theta=\frac{z^{2}}{r^{2}} & =\frac{z^{2}}{x^{2}+y^{2}+z^{2}} \\
= & \frac{\left(l a_{0}\right)^{2}}{\left(m a_{0}\right)^{2}+\left(n a_{0}\right)^{2}+\left(l a_{0}\right)^{2}} \\
= & \frac{l^{2}}{m^{2}+n^{2}+l^{2}} \\
|\vec{r}|^{3}=\left(x^{2}+y^{2}+z^{2}\right)^{3 / 2}= & {\left[\left(m a_{0}\right)^{2}+\left(n a_{0}\right)^{2}+\left(l a_{0}\right)^{2}\right]^{3 / 2} } \\
& =a_{0}^{3}\left(m^{2}+n^{2}+l^{2}\right)^{3 / 2} .
\end{aligned}
$$

Incorporating Eqs. (3) and (4) into Eq. (2), the final expression is derived for the $z$ component of the magnetic field $B_{z}$ from a ferromagnetic sphere at the atomic site with indices $(m, n, l)$

$$
\begin{aligned}
B_{Z}(m, n, l)= & \frac{M_{0}\left(2 l^{2}-m^{2}-n^{2}\right)}{a_{0}^{3}\left(m^{2}+n^{2}+l^{2}\right)^{5 / 2}} \\
& m=\{167,168, \ldots,+\infty\} \\
& n=\{-\infty, \ldots,-1,0,+1, \ldots,+\infty\} \\
& l=\{-\infty, \ldots,-1,0,+1, \ldots,+\infty\}
\end{aligned}
$$

The index range for the $x$ axis starts with the integer 167, since expression (5) was derived for the $50 \mathrm{~nm}$ radius ferromagnetic sphere (166.66 times the lattice parameter $a_{0}$ $=3 \AA$ ) at the center of the coordinate system.

\section{MAGNETIC RESONANCE DIFFRACTION CALCULATION RESULTS}

An interesting prediction is made from the model when numerical summation is computed for the histogram of the number of resonant spin sites within a $1-G$ wide shell of constant $B_{z}$, as shown in Fig. 2(a). This value of the bin width is selected since the linewidth broadening in solids is of the order of $1 \mathrm{G}^{30}$ In the field range between approxi- 


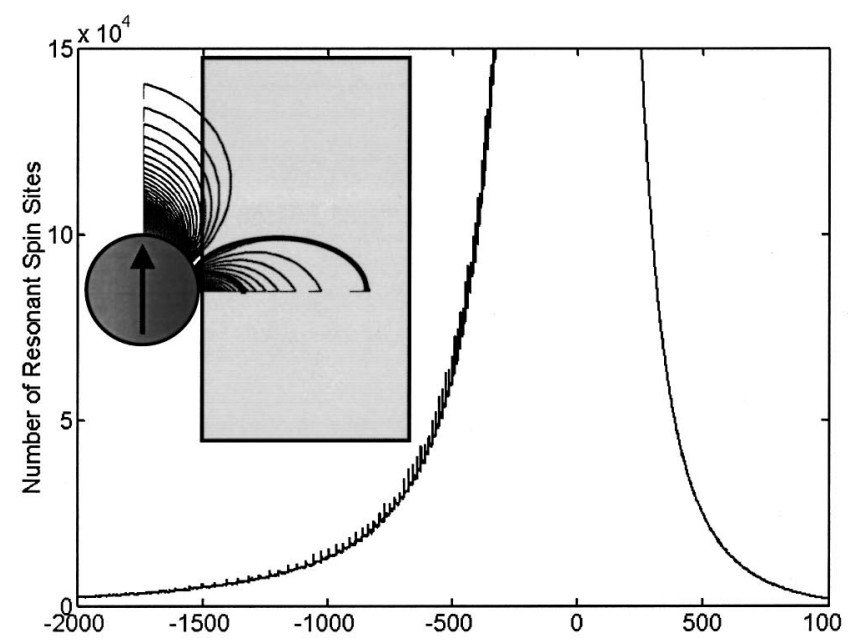

(a)

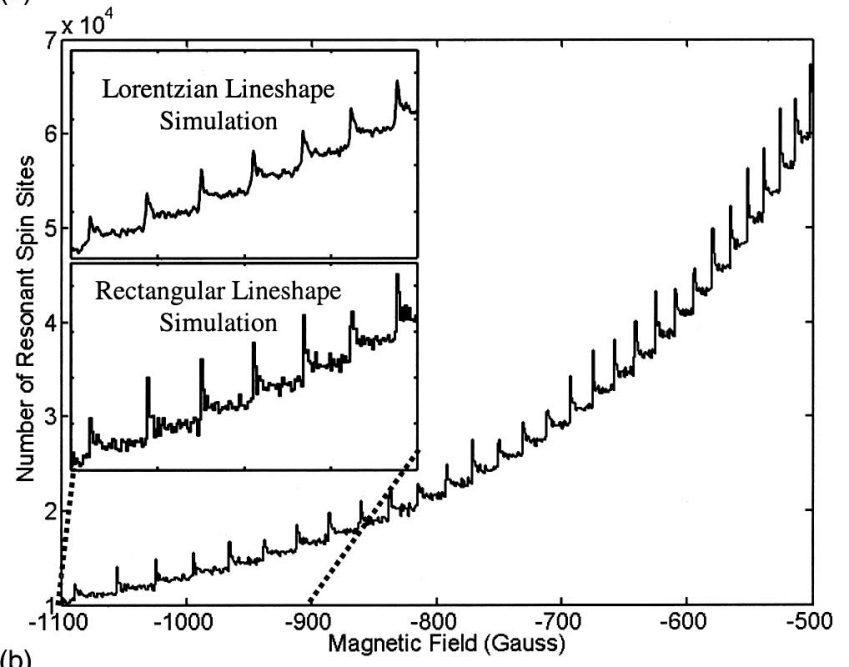

(b)

FIG. 2. Histogram of the number of resonant spin sites of the $3-\AA$ unit-cell size simple cubic crystal in the presence of a large polarizing field $B_{0}$ and the field from a $100 \mathrm{~nm}$ diameter cobalt ferromagnetic sphere. Magnetic resonance spectrum shows distinct peaks in the negative range of magnetic field from a ferromagnetic sphere. Enhanced contours of constant $B_{Z}$ from the sphere between which the peaks occur are indicated in the inset of the figure. The lower part of the figure shows the enlarged version of the spectrum between the values of $B_{0}-1100 \mathrm{G}$ and $B_{0}-500 \mathrm{G}$. Inset shows the difference between the calculations using a $1-G$ wide rectangular-shaped spectral response for the spin vs the more experimentally realistic but more computationally time consuming $1-\mathrm{G}$ linewidth Lorentzian spectral response. Although there is a slight broadening of the magnetic resonance diffraction peaks in the Lorentzian linewidth case, the locations of the spectral peaks remain unchanged and provide sufficient spectral peak contrast.

mately $B_{0}-1800 \mathrm{G}$ and $B_{0}-200 \mathrm{G}$, distinct peaks occur in the histogram of the number of resonant spin sites, while there are no distinguishable features in the positive magnetic field range. A magnified view of the spectral region between $B_{0}-1100 \mathrm{G}$ and $B_{0}-500 \mathrm{G}$ is shown in Fig. 2(b). It is at first surprising to find that at certain magnetic field values there are approximately $10 \%-20 \%$ more resonant spin sites than for the adjacent magnetic field values. It is emphasized that the appearance of the magnetic resonance spectral peaks is the direct signature of the discrete atomic lattice sites. Magnetic resonance of a continuous medium would result in the monotonic spectrum on the positive and negative values of the magnetic field.
In this calculation, and all the subsequent results in this article, a rectangular-shaped spectral response for the spin was used in order to reduce the complexity of the computation. A more experimentally realistic calculation would assume a Lorentzian linewidth spectral response, which would require a significant increase in the computational time. The inset of Fig. 2(b) shows the difference between the calculations for the 1-G wide rectangular-shaped spectral response for the spin versus a 1-G linewidth Lorentzian spectral response in the field range from $B_{0}-1100 \mathrm{G}$ to $B_{0}-900 \mathrm{G}$. Although there is a slight broadening of the magnetic resonance diffraction spectral peaks for the Lorentzian linewidth case, the locations of the magnetic resonance diffraction peaks remain unchanged, and the spectral peaks continue to be very distinguishable from the background signal, thereby providing the required spectral peak contrast. It is also noted here that linewidth narrowing techniques in solid state NMR are not being considered in this article. Introduction of such techniques to this magnetic resonance diffraction method might provide a further spectral peak contrast.

A clear explanation for the appearance of the magnetic resonance spectral peaks emerges when one visualizes in $3 \mathrm{D}$ the resonant spins under the influence of the polarizing magnetic field $B_{0}$ and the magnetic field from a ferromagnetic sphere. Figure 3 shows five such representation plots for crystal lattice spin sites that are in resonance at a sequence of five magnetic-field values. Only the positive values for the $y$-axis indices are plotted for clarity. The magnetic field bin size is $1 \mathrm{G}$, and the sequence is centered at the magnetic field value of $B_{0}-1025 \mathrm{G}$, the location of one of the sharp resonant peaks in the spectrum of Fig. 2. At the two lower magnetic field values of $B_{0}-1027 \mathrm{G}$ and $B_{0}-1026 \mathrm{G}$, there are two empty regions at the top and bottom sections of the $1-\mathrm{G}$ thin shell of constant $B_{z}$ where no atomic spin sites are intersected. The reason is that at these magnetic field values, the top and bottom sections of the shell of constant $B_{z}$ from a ferromagnetic sphere are between the two lattice planes, and do not intersect the atomic layers. However, there are still many atoms of the crystal shown in Figs. 3(a) and 3(b) that satisfy the resonance condition, and they form the background signal in the magnetic resonance spectra of Fig. 2. At the field value of $B_{0}-1025 \mathrm{G}$ the shell of constant $B_{z}$ intersects the crystal lattice so that a large number of spin sites from the two lattice planes at the top and bottom sections of the resonant shell satisfy the resonance condition. The two bands of the resonant atoms from the lattice planes are clearly visible in Fig. 3(c), as indicated by the arrows in the plot, and these are the resonant rings that are responsible for the sharp peaks in the magnetic resonance spectrum. At the next higher values of the magnetic field, $B_{0}-1024 \mathrm{G}$ and $B_{0}-1023 \mathrm{G}$, these rings of resonant spin sites from the lattice planes slowly disappear as the top and bottom sections of the resonant shell of constant $B_{z}$ move between the next two adjacent atomic lattice planes. The inset of Fig. 2(a) indicates the resonant shells of constant $B_{z}$ with the lowest and highest magnetic field value in the spectra where the diffraction peaks occur. The spin sites between these border shells of constant $B_{z}$ are probed in this method of magnetic resonance diffraction. 


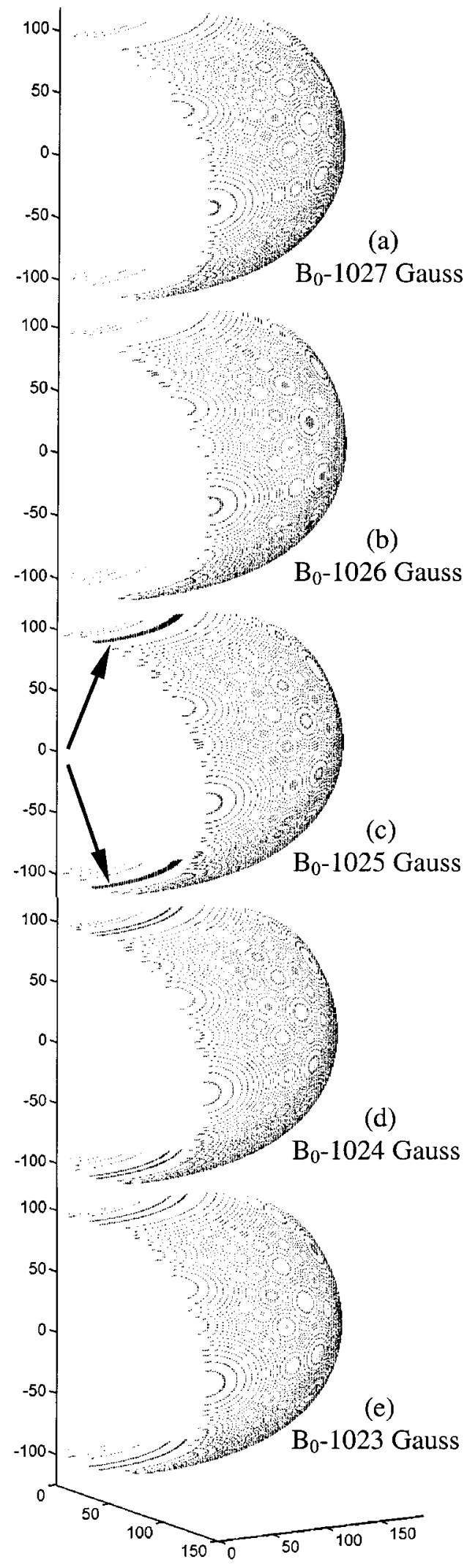

FIG. 3. Three-dimensional visualization plots of the simultaneously resonant spin sites of the crystal lattice. The images are centered at the magnetic field value of $B_{0}-1025 \mathrm{G}$, the location of one of the spectral peaks in the spectrum of Fig. 2. At this magnetic field value, the top and bottom sections of the shell of constant $B_{Z}$ intersect the two atomic lattice planes, as indicated by the arrows. These rings of resonant atoms are responsible for the magnetic resonance diffraction peaks. At the lower and higher values of the magnetic field, the top and bottom sections of the shell of constant $B_{Z}$ are between atomic lattice planes, thus no peaks appear in the spectrum.
Magnetic resonance diffraction using the magnetic field from a ferromagnetic sphere thus results in distinct peaks in the spectra that reveal the underlying atomic structure, although this is accomplished using a different method than the one originally proposed by Mansfield and Grannel. As opposed to using linear magnetic field gradients in resolving the crystal planes, ferromagnetic sphere provides highly nonlinear magnetic field gradients. Nevertheless, as Fig. 3 shows, the shells of constant $B_{z}$ intersect the crystal in a way that allows one to resolve and reveal the underlying atomic lattice planes in the magnetic resonance signal. For the case shown in Fig. 3, the shells of constant $B_{z}$ intersect the $\langle 100\rangle$ planes of the crystal to give the diffraction peaks. This intersection of the shells of constant $B_{z}$ from a ferromagnetic sphere through the crystal lattice draws a strong analogy to the Ewald construction in crystallography. ${ }^{27}$ However, one has to remember that in magnetic resonance diffraction using ferromagnetic spheres, the intersection of the shells of constant $B_{z}$ occurs in the real space lattice, while the Ewald construction is carried out in the reciprocal lattice space.

Reasons for the appearance of magnetic resonance peaks of Fig. 2 in the configuration of Fig. 1 also reveal why such peaks were not predicted or observed in all the MRFM investigations to date. The samples in reported MRFM work are normally positioned so that the magnetic moment of the ferromagnetic particle is perpendicular to the sample surface. This conventional configuration is described in the inset of Fig. 4(a). It is not surprising that this choice of experimental conditions is made, since the magnetic field gradient along the $z$ axis in such a configuration is twice as large as the gradient along the $x$ axis in the configuration of Fig. 1. However, by considering the intersections of shells of constant $B_{z}$ and the crystal lattice that result in the diffraction peaks, as shown in the inset of Fig. 2(a), one realizes that none of the shells can intersect the crystal in Fig. 1(a) in a way that would resolve and reveal the underlying atomic lattice planes. Figure 4(a) shows the simulation for the configuration shown in the inset of Fig. 4(a), and one observes no clear signatures of the atomic lattices present.

Although peaks are not evident for a crystal rotated at the angle of $90^{\circ}$ angle around the $y$ axis, as was shown in Fig. 4(a), much like in other crystallographic methods, the spectral peaks are expected at other angles where the thin azimuthally symmetric resonant shells of constant $B_{z}$ properly intersect the crystal lattice planes. In the azimuthally symmetric system of Fig. 1, there is no diffraction spectra dependence for crystal rotation around the $z$ axis. However, there is an angular dependence of the diffraction spectra on the crystal rotation around the $x$ axis by the angle $\phi$, as well as on the rotation of the crystal with respect to the $y$ axis by the angle $\theta$. Two separate examples are given for the rotation of the crystal by $45^{\circ}$ around the $y$ axis in Fig. 4(b) and by the same angle around the $x$ axis in Fig. 4(c). As expected, the magnetic resonance diffraction peaks appear since the shells of constant $B_{z}$ properly intersect the atomic lattice planes at appropriate magnetic field values. Drawing on the analogy with other crystallographic techniques, the shells of constant $B_{z}$ intersect the $\langle 110\rangle$ and $\langle 101\rangle$ planes of the crystal for the two cases shown in Figs. 4(b) and 4(c). It should be men- 


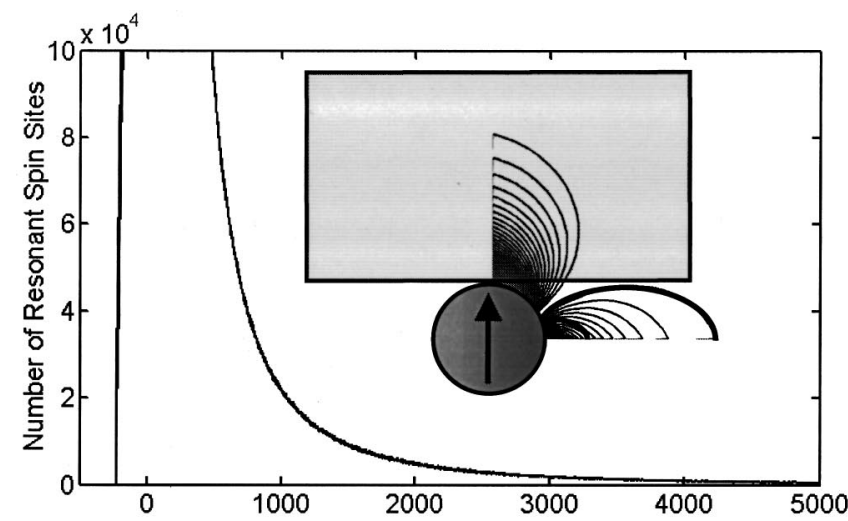

(a)

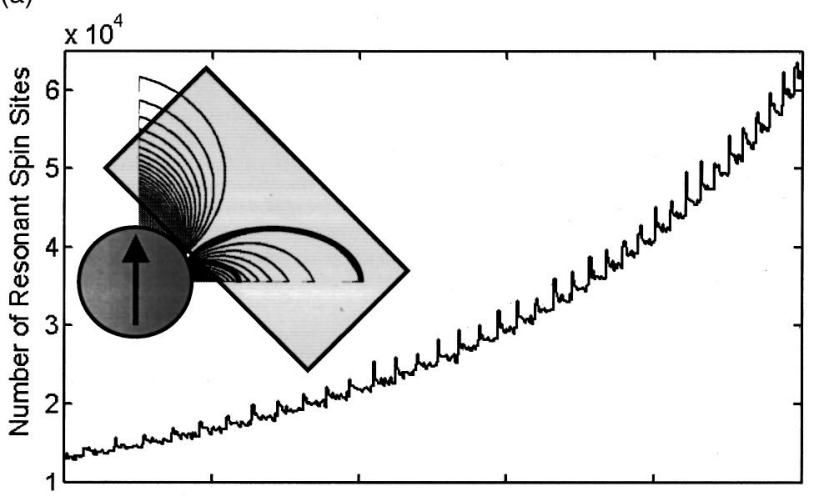

(b)

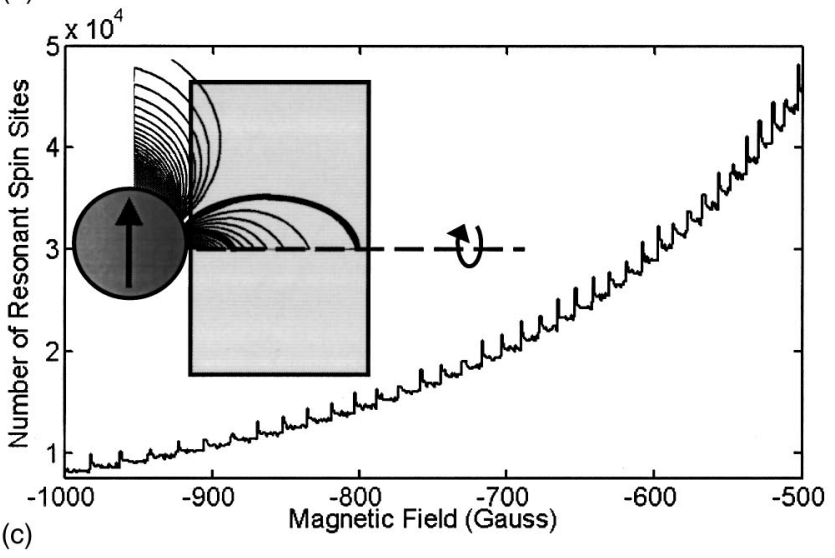

FIG. 4. Magnetic resonance spectra for three different angle configurations. In (a), the polarizing magnetic field is applied perpendicular to the sample surface. The shells of constant $B_{Z}$ responsible for obtaining sharp magnetic resonance spectral peaks do not intersect the sample, and no peaks occur. At the sample rotation of $45^{\circ}$ around the $y$ axis in (b), the shells of constant $B_{Z}$ reveal new spectral peaks by intersecting different atomic lattice planes. Rotation of the sample around the $x$ axis in (c) by $45^{\circ}$ again results in magnetic resonance spectral peaks.

tioned that the angular resolution of the sharpness of spectral peaks was also computed, and the linewidth of the angular resolution was found to be approximately $\Delta=0.5^{\circ}$ for the $y$ axis of rotation, and $\Delta=0.2^{\circ}$ for the $x$ axis of rotation.

The dependence of the spectral peaks on the crystal lattice dimensions and the ferromagnetic sphere size and magnetization was also investigated. The spectra for the five simple cubic crystal lattices with the unit-cell size ranging from $a_{0}=2.8$ to $3.2 \AA$ is shown in Fig. 5. There is a distinguishable difference among the five spectra, with different frequencies of the magnetic resonance peaks for each spe-



FIG. 5. Magnetic resonance spectra from simple cubic crystals with different unit-cell dimensions. Different crystal dimensions exhibit different spectral peaks frequencies, providing a method for obtaining structural parameters of the crystals.

cific unit-cell size. This feature is significant for potential crystallography applications, since the different frequencies of the method provide a measurement scale for distinguishing different crystal lattice dimensions. The variation in the ferromagnetic sphere size also results in noticeable differences in the observed magnetic resonance spectra. Figure 6 shows the spectra for the three different cobalt spheres with diameters of 50, 100, and $200 \mathrm{~nm}$. One observes that the frequency of the magnetic resonance spectral peaks is proportional to the diameter of the ferromagnetic sphere, as is the number of resonant spins in each spectral peak. This observation suggests an important experimental trade off, with smaller spheres providing higher spectral resolution, but with the requirement of higher experimental sensitivity due to the smaller number of spins that needs to be detected. Larger ferromagnetic spheres provide lower spectral resolution, but allow less stringent sensitivity requirements due to the higher number of spins that needs to be detected in each spectral peak. Simulations of the effect of sphere moment also reveal that higher moment materials provide more numerous and sharper peaks at higher field values than lower moment spheres. Therefore, cobalt or iron spheres are preferred over the lower saturation magnetization materials such as nickel.

Further interesting applications of the magnetic resonance diffraction using ferromagnetic spheres can be pursued with a closer analysis of the resonant spin sites within a thin shell of constant magnetic field $B_{z}$, as shown in Fig. 3. It is apparent that the spectral diffraction peaks come from the very narrow regions of the sample, while there is a large background signal from other resonant spin sites that are intersected by the $1-\mathrm{G}$ thick shell of constant $B_{z}$. This feature of magnetic resonance diffraction can be exploited in the studies of crystalline samples that are different from the semi-infinite crystals of Fig. 1. The first example given is a simple cubic crystalline film with a thickness of 100 unit cells in the $x$ direction, but infinite in the $y$ and $z$ directions, and the spectrum expected from such a structure is shown in 




(a)

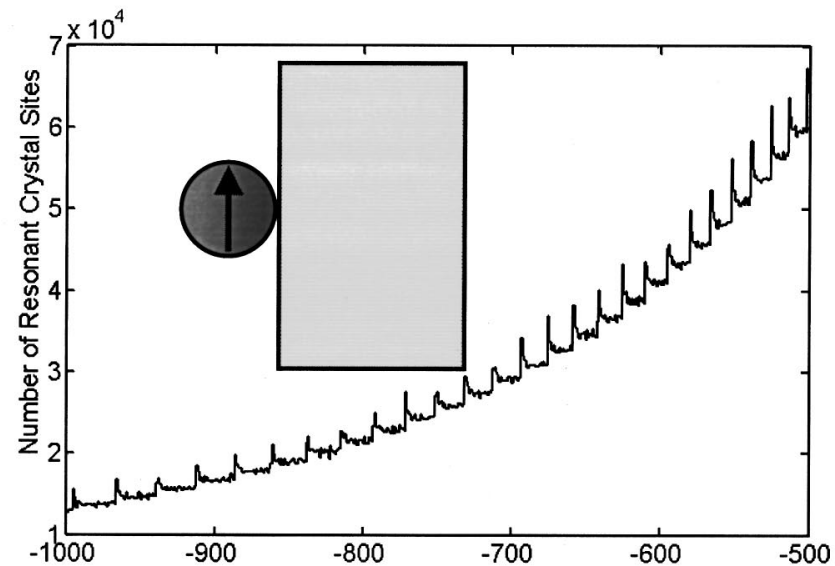

(b)



FIG. 6. Magnetic resonance spectra from the simple cubic crystal and three different cobalt sphere dimensions. Higher spectral resolution for the $50 \mathrm{~nm}$ diameter cobalt sphere in (a) is offset by the lower number of resonant spin sites that needs to be detected. Larger number of resonant spin sites in (c) for the $200 \mathrm{~nm}$ diameter cobalt sphere is accompanied by the lower spectral resolution.

Fig. 7. The resonant peaks occur at the same location as for the semi-infinite crystal of Fig. 1, but they lack the large background signal because there are no atoms intersected by the resonant magnetic field shell beyond the 100th unit cell. This reduction in the large background signal would most certainly be advantageous in experimental work, and would qualify this magnetic resonance diffraction technique for use in thin film studies. The same conclusions from the semi-



FIG. 7. Magnetic resonance spectra from a thin simple cubic crystal film. The film has 100 unit cells in the $x$ direction, with the unit-cell size of $3 \AA$. Large background signal of Fig. 2 is reduced, since no spins are detected beyond the 100th cell in the $x$ direction.

infinite crystal regarding the angular resolution and dependence of spectral peaks on the crystal unit-cell size apply for the thin film crystal case.

In addition to the thin crystalline structures, the fact that only narrow sections of the sample contribute to the peaks in the magnetic resonance spectra could be applied to the studies of small crystallites. Figure 8 shows a simulation of a $100 \times 100 \times 100$ atoms cubic lattice crystallite scanned by the $100 \mathrm{~nm}$ diameter cobalt sphere. As the ferromagnetic sphere is scanned over the sample in $12 \mathrm{~nm}$ increments, the spectrum shows resonant diffraction peaks in the narrow scan range of approximately $50 \mathrm{~nm}$ where the atomic lattice planes of the crystallite pass through the resonant slices of constant $B_{z}$. A sequence of spectra shows, as in the thin film case, that the large background signal is almost nonexistent in the magnetic resonance spectra. Therefore, small crystallites would also provide distinct peaks in magnetic resonance spectra with many spins in the atomic lattice planes coherently contributing to the signal.

\section{PROPOSAL FOR EXPERIMENTAL DEMONSTRATION}

Following the description of the magnetic resonance diffraction using the field from a ferromagnetic sphere, a discussion on the potential experimental techniques that are suitable for the confirmation of the theory described in this article is in order. The main argument in this article lies in the finding that this magnetic resonance diffraction method provides an opportunity to detect, using a slightly reconfigured form of MRFM, the presence of atomic lattice planes by detecting the numerous spins that are coherently in resonance at the same value of the magnetic field. This significantly relaxes the experimental constraints on the measurement from the single-spin detection proposals of MRFM, since a larger detected signal is available from additional spins. This method also alleviates potential single-spin detection complications ${ }^{31}$ in MRFM. Furthermore, the size of the ferromagnetic sphere that would provide sufficient spectral resolution can be an order of magnitude larger than the one believed to be necessary ${ }^{14}$ for single-spin detection, further 

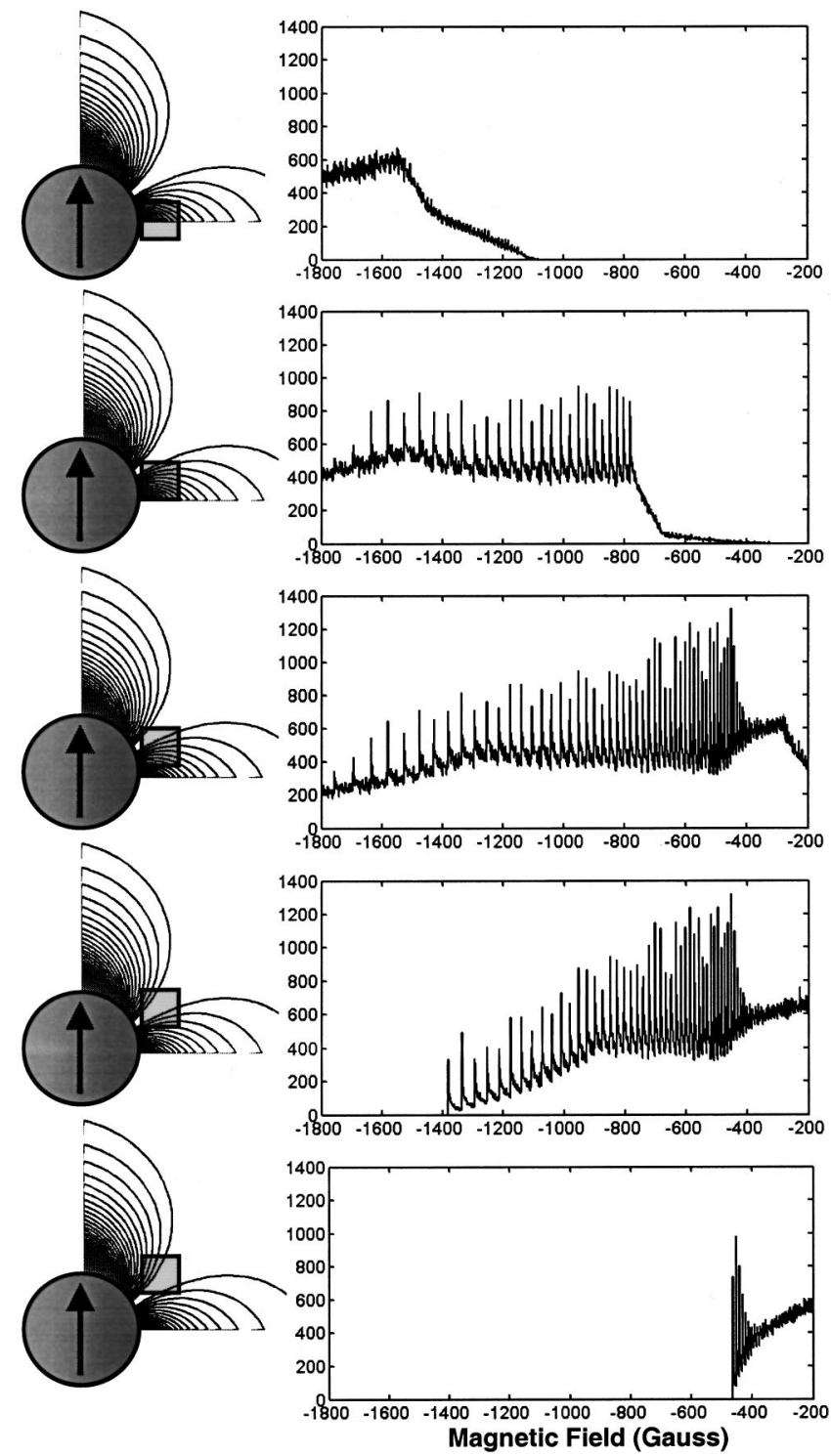

FIG. 8. Magnetic resonance spectra from a $100 \times 100 \times 100$ atoms crystallite with the simple cubic structure and $3-\AA$ unit-cell size. The crystallite is scanned under the $100 \mathrm{~nm}$ cobalt ferromagnetic sphere in $12 \mathrm{~nm}$ increments. As the cube passes through the resonant shells of constant $B_{Z}$, atomic lattice planes of the crystallite are resonant at various field values and magnetic resonance diffraction peaks appear.

easing experimental realization of the technique. The number of spins that have to be detected in magnetic resonance diffraction using ferromagnetic sphere ranges between $10^{4}$ and $10^{5}$ and the number of spins in a spectral peak above the background level is on the order of $10^{3}$, as Fig. 2 shows. With the current sub-attonewton force detection capability using an ultra-thin cantilever ${ }^{32}$ and sensitive fiber-optic interferometer, ${ }^{33}$ as well as the availability of the ultra-high magnetic field gradient sources, ${ }^{21-23}$ the experimental groundwork is already laid out for the detection of magnetic resonance diffraction of nuclear spins using a ferromagnetic sphere mounted on a mechanical cantilever.

Although this magnetic resonance diffraction method significantly relaxes the technical requirements in MRFM for the detection of atomic planes by detecting between $10^{3}$ and $10^{5}$ spins, the technique proposed in this article still faces experimental challenges. In most room-temperature NMR experiments the fractional polarization is quite small, on the order of $10^{-5}$, and improves at helium temperatures to values on the order of $10^{-3}$. This will have important implications for observing the magnetic resonance diffraction peaks at those temperatures due to the significant spin-noise background. However, cantilever operation at millikelvin temperatures has been demonstrated, ${ }^{32}$ and similar technical advancements might alleviate the spin-noise problem. The spin-noise problem in magnetic resonance is a topic of ongoing investigations and still somewhat controversial and not well understood. ${ }^{34,35}$ Additionally, this article has focused on cobalt as the material of choice, but magnetic materials with similar saturation magnetization but higher anisotropy, such as rare-earth alloy $\mathrm{PrFeB}$, may be needed to reduce thermal fluctuations of the sphere probe..$^{22,36}$

It should be emphasized that although this article has been aimed at the MRFM community for the realization of magnetic resonance diffraction, many other detection systems may be potential candidates. They include micro-coil $\mathrm{NMR},{ }^{37}$ micro-superconducting quantum interference device detectors, ${ }^{38}$ Hall sensors, ${ }^{39}$ superconducting resonators, ${ }^{40}$ and microwave waveguides, ${ }^{41}$ and these provide additional routes to the proposed atomic resolution magnetic resonance diffraction. There are many further questions to be pursued in the field of magnetic resonance diffraction using the magnetic field from a ferromagnetic sphere. They include the diffraction spectra from crystal structures other than the simple cubic lattice discussed in this article, the dependence of the spectra on ferromagnetic particle shapes other than the sphere, and the dependence of the diffraction peaks on various intrinsic line shapes and widths. These topics are the subject of an ongoing investigation and will be published elsewhere. Although this article is focused on imaging and crystallographic applications, there are intriguing parallels between this diffraction technique and other scientific topics where the interaction of the nanoscopic ferromagnetic objects and discrete spins of the crystal lattice are of interest, most notably in the field of quantum computation. ${ }^{42-45}$

\section{ACKNOWLEDGMENTS}

This work was made possible by the unconditional support and financial assistance of Professor Axel Scherer of California Institute of Technology. The author also thanks Willie Padilla and David Schurig of University of CaliforniaSan Diego for computational help, and Joyce Wong for careful reading of the manuscript. Part of this work was conducted while the author was with the Department of Physics, University of California-San Diego.

${ }^{1}$ P. C. Lauterbur, Nature (London) 242, 190 (1973).

${ }^{2}$ P. Mansfield and P. K. Grannell, J. Phys. C 6, L422 (1973).

${ }^{3}$ F. W. Wehrli, Prog. Nucl. Magn. Reson. Spectrosc. 28, 87 (1995).

${ }^{4}$ J. Aguayo, S. Blackband, J. Schoeniger, M. Mattingly, and M. Hintermann, Nature (London) 322, 190 (1986).

${ }^{5}$ S. C. Lee et al., J. Magn. Reson. 150, 207 (2001).

${ }^{6}$ P. T. Callaghan, Principles of Nuclear Magnetic-Resonance Microscopy (Oxford University, New York, 1991).

${ }^{7}$ A. Abragam, Principles of Nuclear Magnetism (Oxford University, New York, 1983). 
${ }^{8}$ Y. Takai, T. Kawasaki, Y. Kimura, T. Ikuta, and R. Shimizu, Phys. Rev. Lett. 87, 106105 (2001).

${ }^{9}$ G. Binning, H. Rohrer, C. Gerber, and E. Weibel, Phys. Rev. Lett. 49, 57 (1982).

${ }^{10}$ G. Binning, C. F. Quate, and C. Gerber, Phys. Rev. Lett. 56, 930 (1986).

${ }^{11}$ R. Wiesendanger, H. J. Guntherodt, G. Guntherodt, R. J. Gambino, and R. Ruf, Phys. Rev. Lett. 65, 247 (1990).

${ }^{12}$ Y. Manassen, R. J. Hamers, J. E. Demuth, and A. J. Castellano, Jr. Phys. Rev. Lett. 62, 2531 (1989).

${ }^{13}$ J. A. Sidles, Appl. Phys. Lett. 58, 2854 (1991).

${ }^{14}$ J. A. Sidles et al., Rev. Mod. Phys. 67, 249 (1995).

${ }^{15}$ J. A. Sidles and D. Rugar, Phys. Rev. Lett. 70, 3506 (1993)

${ }^{16}$ D. I. Hoult and R. E. Richards, J. Magn. Reson. 24, 71 (1976).

${ }^{17}$ D. I. Hoult and P. C. Lauterbur, J. Magn. Reson. 34, 425 (1979).

${ }^{18}$ D. Rugar, C. S. Yannoni, and J. A. Sidles, Nature (London) 360, 563 (1992).

${ }^{19}$ D. Rugar et al., Science 264, 1560 (1994).

${ }^{20}$ Z. Zhang, P. C. Hammel, and P. E. Wigen, Appl. Phys. Lett. 68, 2005 (1996).

${ }^{21}$ K. J. Bruland, W. M. Doughety, J. L. Garbini, J. A. Sidles, and S. H. Chao, Appl. Phys. Lett. 73, 3159 (1998)

${ }^{22}$ B. C. Stipe, H. J. Mamin, T. D. Stowe, T. W. Kenny, and D. Rugar, Phys. Rev. Lett. 86, 2874 (2001).

${ }^{23}$ T. D. Stowe et al., Appl. Phys. Lett. 71, 288 (1997).

${ }^{24}$ O. Zuger and D. Rugar, Appl. Phys. Lett. 63, 2496 (1993).

${ }^{25}$ O. Zuger, S. T. Hoen, C. S. Yannoni, and D. Rugar, J. Appl. Phys. 79, 1881 (1996).

${ }^{26}$ P. Mansfield and P. K. Grannell, Phys. Rev. B 12, 3618 (1975).

${ }^{27}$ B. D. Cullity, Elements of X-ray Diffraction (Addison-Wesley, Boston, MA, 2001).
${ }^{28}$ G. E. Bacon, Neutron Diffraction (Clarendon, Oxford, 1962).

${ }^{29}$ J. C. H. Spence and J. M. Zuo, Electron Microdiffraction, (Kluwer Academic, New York, 1992).

${ }^{30}$ C. P. Slichter, Principles of Magnetic Resonance (Springer-Verlag, New York, 1996)

${ }^{31}$ G. P. Berman and V. I. Tsifrinovich, Phys. Rev. B 61, 3524 (2000).

${ }^{32}$ H. J. Mamin and D. Rugar, Appl. Phys. Lett. 79, 3358 (2001).

${ }^{33}$ D. Rugar, H. J. Mamin, and P. Guethner, Appl. Phys. Lett. 55, 2588 (1989).

${ }^{34}$ D. I. Hoult and N. S. Ginsberg, J. Magn. Reson. 148, 182 (2001).

${ }^{35}$ J. A. Sidles, J. L. Garbini, W. M. Dougherty, and S. H. Chao, e-print quant-ph/0004106 (http://xxx.lanl.gov)(2001).

${ }^{36}$ J. D. Hannay, R. W. Chantrell, and D. Rugar, J. Appl. Phys. 87, 6827 (2000).

${ }^{37}$ D. L. Olson, T. L. Peck, A. G. Webb, R. L. Magin, and J. V. Sweedler, Science 270, 1967 (1995).

${ }^{38}$ L. R. Narasimhan, C. K. N. Patel, and M. B. Ketchen, IEEE Trans. Appl. Supercond. 9, 3503 (1999).

${ }^{39}$ G. Boero, P. A. Besse, and R. Popovic, Appl. Phys. Lett. 79, 1498 (2001).

${ }^{40}$ R. D. Black et al., Science 259, 793 (1993).

${ }^{41}$ S. Zhang, S. A. Oliver, N. E. Israeloff, and C. Vittoria, Appl. Phys. Lett. 70, 2756 (1997).

${ }^{42}$ G. P. Berman, G. D. Doolen, P. C. Hammel, and V. I. Tsifrinovich, Phys. Rev. B 61, 14694 (2000).

${ }^{43}$ G. P. Berman, G. D. Doolen, P. C. Hammel, and V. I. Tsifrinovich, Phys. Rev. Lett. 86, 2894 (2001).

${ }^{44}$ F. Yamaguchi and Y. Yamamoto, Appl. Phys. A: Mater. Sci. Process. 68, 1 (1999).

${ }^{45}$ J. R. Goldman, T. D. Ladd, F. Yamaguchi, and Y. Yamamoto, Appl. Phys. A: Mater. Sci. Process. 71, 11 (2000). 